\title{
Minimum Weighted Residual Methods in Endogenous Growth Models
}

by

\author{
Michal Kejak*
}

May 2000

*CERGE-EI; Politickych veznu 7; CZ-111 21 Prague, Czech Republic; Phone: $+(420)$ $2240 \quad 05$ 186; Fax: +(420) 224211 374; E-mail: Michal.Kejak@cerge.cuni.cz; http://home.cerge.cuni.cz/mkej 


\begin{abstract}
The paper deals with the application of Minimum Weighted Residual Methods (MWR) in intertemporal optimizing models of endogenous economic growth. In the first part of the paper the basics of the MWR method are described. Attention is mainly concentrated on one special class of MWR methods: the orthogonal collocation method with the Chebyshev polynomial basis. The second part of the paper is devoted to the setup of a model of endogenous growth with human capital accumulation and the government sector and to the derivation of first order conditions which form a Two-Point-Boundary-Value problem. A transformation of the problem which eliminates the growth in variables is then presented and the MWR method is used to solve the model for some policy experiments.
\end{abstract}




\section{Introduction}

Much of the current research in macroeconomics and mainly in neoclassical growth models is based on the intertemporal optimizing infinite-lived representative agent model. Solving these models via derivation of the first order necessary conditions by means of the Pontryagin Maximum Principle or the Bellman dynamical programming approach, we face the so called two point boundary value (TPBV) problem which is much more difficult to solve than the Cauchy initial value (CIV) problem. It is well known that analytical solutions exist only for a very special class of nonlinear problems and for LQ problems or linearized ones.

In situations when we cannot/do not want to limit ourselves to the above special cases, we have to use general numerical methods such as the shooting method or the relaxation method to obtain a solution of the model. Fortunately, a favourable feature of the infinite optimizing problems is the fact that if both the criterion function and the dynamic constraints are autonomous (and equilibrium is saddle path stable), the control variables can be characterized as static (t-invariant) policy functions (or feedback rules) of the state variables. This property is critical for the possibility of using the following methods to aproximate the policy functions: the perturbation method, the time elimination method and minimum weighted residual (or projection) methods. The time elimination method converts the TPBV problem to the CIV problem and, by applying numerical integration starting at a steady state, yields the approximation of policy functions (Mulligan and Sala-i-Martin [1993]). Thus, the approximation is given in the form of "trajectories" of the control variables as functions of the state variables. On the other hand, the perturbation and MWR methods provide a solution in the form of a polynomial approximation. The advantage of such a solution is its analytical form. More specifically, the perturbation method makes use of the Taylor series or the Pade approximations of policy functions in 
a steady state. MWR (or projection) methods (Fletcher [1988] and Judd [1992] ${ }^{1}$ ) approximate the policy functions at some predetermined interval which makes them a good approximation not only at the steady state, as it is in the case of the perturbation method, but in the whole interval.

\section{Minimum Weighted Residual Methods}

To explain the basic idea of MWR methods, suppose that the model is represented by a system of differential equations. First, we have to transform the problem to the form $\mathcal{N}(\mathbf{p})=\mathbf{0}$ where $\mathcal{N}$ is an operator and the function $\mathbf{p}$ is a zero of operator $\mathcal{N}$ which means that $\mathbf{p}$ solves the given system of differential equations. For initial value problems, a zero $\mathbf{p}$ of operator $\mathcal{N}$ is a vector function of time; for boundary value problems (which are characteristic for growth models) with $n$ states and $m$ control variables, a zero $\mathbf{p}$ of operator $\mathcal{N}$ is a vector of policy functions which are functions of state variables only, $\mathbf{p}: R^{n} \rightarrow R^{m}$

To determine a specific type of the MWR method, we have to choose the polynomial basis approximating vector function $\mathbf{p}$ and the weighted residual inner product approximating operator $\mathcal{N}$. In this paper I will restrict myself to the simplest MWR method, the orthogonal collocation method with the Chebyshev polynomial basis.

Thus, the solution of a steady TPBV problem with $n$ state variables and $m$ control variables expressed in the form of $m$ policy functions of $n$ state variables can be approximated by the formula

$$
\mathbf{p}(\mathbf{x}) \approx \hat{\mathbf{p}}(\mathbf{x} ; \mathbf{A})=\mathbf{A} \psi(\mathbf{x})=\sum_{i=1}^{q} \mathbf{a}_{i}^{T} \psi_{i}(\mathbf{x})
$$

where $(m \times q)$ parameter matrix $\mathbf{A}=\left(\mathbf{a}_{1}^{T}, \mathbf{a}_{2}^{T}, \ldots, \mathbf{a}_{m}^{T}\right)^{T}$ is a $m$-dimensional column of $q$-dimensional rows of coefficients $\mathbf{a}_{i}^{T}=\left(a_{i 1}, a_{i 2}, \ldots, a_{i q}\right)$. The multidimensional Cheby-

\footnotetext{
${ }^{1}$ See also an excellent book on numerical methods in economics, Judd (1998).
} 
shev basis of $q$ polynomials $\boldsymbol{\psi}(\mathbf{x})=\left(\psi_{1}(\mathbf{x}), \psi_{2}(\mathbf{x}), \ldots, \psi_{q}(\mathbf{x})\right)^{T}$ for our $(n, m)$ problem is obtained by the $n$-fold tensor product $\left.\boldsymbol{\psi}(\mathbf{x})=\phi_{1}\left(x_{1}\right) \otimes \phi_{2}\left(x_{2}\right) \otimes \cdots \otimes \phi_{n}\left(x_{n}\right)\right)$ of the $n$ one-dimensional Chebyshev bases $\phi_{i}\left(x_{i}\right)$ of degree $q_{i}$ in variable $x_{i}$ where $i=1,2, \ldots, n$ and $q=\prod_{i=1}^{n} q_{i}$.

The approximation of operator $\mathcal{N}$ in the case of the collocation method is given by the calculation of the $q$-dimensional polynomial approximation of the $m$ vector policy function $\hat{\mathbf{p}}$ at $q$ important points $\left\{\mathbf{z}_{k}\right\}_{k=1}^{q}$ where $\mathbf{z}_{k} \in R^{n}$. Typically, these points are zeros of the $q$-dimensional polynomial basis $\boldsymbol{\psi}(\mathbf{x})$, i.e. $\psi_{k}\left(\mathbf{z}_{k}\right)=0$ where $k=1, \ldots, q$. Thus, our modification of the MWR method yields the following $(m \times q)$ matrix residual function

$$
\mathbf{R}(\mathbf{x} ; \mathbf{A}) \equiv(\hat{\mathcal{N}}(\hat{\mathbf{p}}))(\mathbf{x})=\left((\mathcal{N}(\hat{\mathbf{p}}))\left(\mathbf{z}_{1}\right), \ldots,\left(\mathcal{N}\left(\hat{\mathbf{p}}\left(\mathbf{z}_{q}\right)\right)=\mathbf{0}\right.\right.
$$

where $\hat{\mathbf{p}}\left(\mathbf{z}_{k}\right)=\mathbf{A} \boldsymbol{\psi}\left(\mathbf{z}_{k}\right)$ and $k=1, \ldots, q$.

In this way, solving the $(n, m)$ TPBV problem with the degrees of approximation $\left(q_{i}\right)_{i=1}^{n}$ can be transformed into a problem of solving $m \times q$ nonlinear algebraic equations in $m \times q$ unknown coefficients with $q=\prod_{i=1}^{n} q_{i}$.

\section{Application of the MWR Method in An Endoge- nous Growth Model}

For demonstration of the application of the MWR method in economics I will set up an aggregate macroeconomic endogenous growth model with human capital accumulation (Lucas (1988)) and extend it to include the government sector (Kejak (1995)). The economy is populated by identical workers endowed with the same skill level $H$ and infinite lifetime. They devote a fraction $l$ of their (non-leisure) time to current production, and the remaining $1-l$ to human capital accumulation. Thus, the effective labor input in production is $L=l H$. The economy also consists of a large number of identical firms, 
therefore, we assume that the firms behave competitively with the following production function $Y=F(K, H)=K^{\beta} L^{1-\beta} H_{a}^{\gamma}=K^{\beta}(l H)^{1-\beta} H_{a}^{\gamma}$, where $Y$ is the output of the firm, $K$ is physical capital, and the term $H_{a}^{\gamma}$ introduces an "external effect" which is related to the average level of human capital in the economy and $0<\beta<1$ and $\gamma \geq 0$. Because the workers are identical, the average skills in equilibrium coincide with individual skills $\left(H=H_{a}\right)$. There are two factor markets, one for labor and one for capital services. The price of labor is the wage $w_{t}$ and the interest rate $r_{t}$ is the price of capital.

Each agent in the economy wants to maximize his lifetime utility or welfare $V_{0}=$ $\int_{0}^{\infty} u\left(C_{t}\right) e^{-\rho t} d t$ which is the discounted sum of instantaneous utilities $u\left(C_{t}\right)$. Therefore, if the constant relative risk aversion utility function is assumed, i.e. $u(C)=C^{1-\theta} /(1-\theta)$, the whole model of the two sector economy can be set up as a dynamic optimization problem with two control variables - consumption $C_{t}$ and the time devoted to production $l_{t}$ in the following form:

$$
\begin{aligned}
\max _{C_{t}, l_{t}} & V_{0}=\int_{0}^{\infty}\left(\frac{C_{t}^{1-\theta}}{1-\theta}\right) e^{-\rho t} d t \\
\text { s.t. } & \dot{K}_{t}=\left(1-\tau_{K}\right) r_{t} K_{t}+\left(1-\tau_{L}\right) w_{t} l_{t} H_{t}+\tau w_{t}\left(1-l_{t}\right) H_{t}-T_{t}-C_{t}, \quad K_{0}>0(4) \\
\dot{H}_{t} & =\phi\left(1-l_{t}\right) H_{t}, \quad H_{0}>0,
\end{aligned}
$$

where $\tau_{L}$ is the labor income tax rate, $\tau_{K}$ the capital income tax rate, $\tau$ the education subsidy rate, and $T_{t}$ the lump-sum tax. Government expenditure (per capita) is given by the constant fraction of output $G_{t}=\kappa Y_{t}$ and is financed by the net collected taxes $G_{t}=\tau_{K} r_{t} K_{t}+\tau_{L} w_{t} l_{t} H_{t}-\tau w_{t}\left(1-l_{t}\right) H_{t}+T_{t}$. Equations (4) and (5) describe the process of physical and human capital accumulation respectively. A linear Uzawa-Rosen type production function for human capital with the parameter of the effectiveness of investment in human capital $\phi$ is used in the latter equation. Physical capital is depreciated by rate $\delta$ and human capital does not depreciate.

Solving the dynamic optimization problem given in (3)-(5) by using the Pontryagin maximum principle, we can write the current-value Hamiltonian $\mathcal{H}\left(K_{t}, H_{t}, H_{a t} ; C_{t}, l_{t} ; \lambda_{t}, \mu_{t}\right)=$ 
$\frac{C_{t}{ }^{1-\theta}-1}{1-\theta}+\lambda_{t} \dot{K}_{t}+\mu_{t} \dot{H}_{t}$, where $\lambda_{t}$ and $\mu_{t}$ denote the shadow prices (or costate variables) of physical and human capital, respectively. By eliminating the shadow prices $\lambda_{t}$ nd $\mu_{t}$ in the first order conditions, the TPBV problem can be expressed in the form:

$$
\begin{aligned}
\dot{K}_{t}= & (1-\kappa) Y_{t}-C_{t}-\delta K_{t} \\
\dot{H}_{t}= & \phi\left(1-l_{t}\right) H_{t} \\
\dot{C}_{t}= & \sigma\left[\left(1-\tau_{K}\right)\left(\frac{Y_{t}}{\beta K_{t}}-\delta\right)-\rho\right] C_{t} \\
\dot{l}_{t}= & \left\{\left(\tau_{K}-\kappa\right) \frac{Y_{t}}{K_{t}}+\frac{\delta\left(1-\tau_{K}\right)}{\beta}-\delta-\frac{C_{t}}{K_{t}}+\frac{\phi}{\beta}\left[(\gamma-\beta)\left(1-l_{t}\right)+l_{t}+\frac{1-\tau_{L}}{1-\tau_{L}-\tau}\right]\right\} l_{t} \\
& K_{0}>0, \quad H_{0}>0 \\
& \lim _{t \rightarrow \infty} e^{-\rho t} \lambda_{t} K_{t}=0, \quad \lim _{t \rightarrow \infty} e^{-\rho t} \mu_{t} H_{t}=0,
\end{aligned}
$$

where equation (10) specifies the initial values and equation (11) the transversality conditions.

When we want to use the projection method in growth models, and, in principle, any of the methods based on the polynomial approximation of policy functions, i.e. the perturbation method or the time-elimination method, we face the problem that many of the variables of the model exhibit balanced growth rates which exclude the possibility of finding a limited region (a point or even an interval) to which we want to relate our approximation. Therefore, we have to find the transformation that enables us to express the model in transformed variables: control-like and state-like variables (Mulligan and Sala-i-Martin (1993)) which have no growth in steady state. Typically, the transformation reduces the dimensionality of the model which is favourable for the presentation of the dynamic behaviour as well.

Based on the relation between the steady state growth of physical and human capital given by $\left(\frac{\dot{H}_{t}}{H_{t}}\right)_{s s}=\frac{1-\beta}{1-\beta+\gamma}\left(\frac{\dot{K}_{t}}{K_{t}}\right)_{s s}$ and the relation between the steady state growth of physical capital and that of consumption $\left(\frac{\dot{K}_{t}}{K_{t}}\right)_{s s}=\left(\frac{\dot{C}_{t}}{C_{t}}\right)_{s s}$, we can suggest the follow- 
ing transformations ${ }^{2} k_{t} \equiv K_{t} H_{t}^{-\left(1+\frac{\gamma}{1-\beta}\right)}$ and $c_{t} \equiv C_{t} K_{t}^{-1}$ which yield the reduced model equations with zero growth rate in steady state:

$$
\begin{aligned}
\frac{\dot{k_{t}}}{k_{t}} & =(1-\kappa) \frac{F_{K}\left(k_{t}, l_{t}\right)}{\beta}-\delta-c_{t}-\left(1+\frac{\gamma}{1-\beta}\right) \phi\left(1-l_{t}\right) \\
\frac{\dot{c_{t}}}{c_{t}} & =\left(\sigma\left(1-\tau_{K}\right)-\frac{1-\kappa}{\beta}\right) F_{K}\left(k_{t}, l_{t}\right)-\sigma(\delta+\rho)+\delta\left(1+\sigma \tau_{K}\right)+c_{t} \\
\frac{\dot{l_{t}}}{l_{t}} & =\frac{\left(\tau_{K}-\kappa\right)}{\beta} \beta F_{K}\left(k_{t}, l_{t}\right)+\frac{\delta\left(1-\tau_{K}\right)}{\beta}+\frac{\phi}{\beta} \frac{1-\tau_{L}}{1-\tau_{L}-\tau}-\delta-c_{t}+\phi\left(\frac{\gamma}{\beta}-1\right)\left(1-l_{t}\right),
\end{aligned}
$$

where the marginal product of physical capital $F_{K}$ can be expressed as $F_{K}=\beta k^{\beta-1} l^{1-\beta}$.

To solve our problem (3)-(5) with the two control-like variables $c_{t}, l_{t}$ and the one state-like variable $k_{t}$, we have to specify two policy functions $c=p(k)$ and $l=q(k)$, where the functions $p$ and $q$ depend monotonically ${ }^{3}$ on variable $k$. Using the following identities $p^{\prime}\left(k_{t}\right)=\frac{d p\left(k_{t}\right)}{d k_{t}}=\frac{\dot{c}_{t}}{\dot{k}_{t}}$ and $q^{\prime}\left(k_{t}\right)=\frac{d q\left(k_{t}\right)}{d k_{t}}=\frac{\dot{i}_{t}}{\dot{k}_{t}}$, we can define operator $\mathcal{N}$ suitable for the application of the MWR method as

$$
\begin{aligned}
& \left(\mathcal{N}_{1}(p)\right)(k)=p^{\prime}(k) \dot{k}-\dot{c}=0 \\
& \left(\mathcal{N}_{2}(q)\right)(k)=q^{\prime}(k) \dot{k}-\dot{l}=0
\end{aligned}
$$

where equations (12)-(14) should be used for expressing $\dot{k}_{t}, \dot{c_{t}}$ and $\dot{l}_{t}$, respectively.

After the specification of the domain for the approximation $\left[k_{1}, k_{2}\right]$ which should include the steady state value of the state-like variable, our approximations of $p$ and $q$ will be parametrically given by

$$
\begin{aligned}
& \hat{p}\left(k ; \mathbf{a}_{\mathbf{1}}\right)=\sum_{i=1}^{q} a_{1 i} \phi_{i}(\tilde{k}) \\
& \hat{q}\left(k ; \mathbf{a}_{\mathbf{2}}\right)=\sum_{i=1}^{q} a_{2 i} \phi_{i}(\tilde{k}),
\end{aligned}
$$

\footnotetext{
${ }^{2}$ In general, it is not certain whether such a transformation leads to the existence of a reduced form of the model, i.e. whether the model can be expressed only by means of the transformed variables or not.

${ }^{3}$ This monotony follows from the properties of a stable saddle path.
} 
where $\phi_{i}$ is $i$ th Chebyshev polynom, $\tilde{k}$ is linear transformation of the interval $\left[k_{1}, k_{2}\right]$ into $[-1,1]$, and $q$ is the degree of approximation (the number of terms used). From equations (15)-(16) and approximations (17) and (18), the residual functions become

$$
\begin{aligned}
R_{1}\left(k ; \mathbf{a}_{\mathbf{1}}, \mathbf{a}_{\mathbf{2}}\right)= & \hat{p}^{\prime}\left(k ; \mathbf{a}_{\mathbf{1}}\right)\left\{(1-\kappa)\left(\frac{\hat{q}\left(k ; \mathbf{a}_{\mathbf{2}}\right)}{k}\right)^{1-\beta}-\delta-\hat{p}\left(k ; \mathbf{a}_{\mathbf{1}}\right)-\left(1+\frac{\gamma}{1-\beta}\right) \phi(1-l)\right\} k \\
- & \left\{\left(\sigma \beta\left(1-\tau_{K}\right)+\kappa-1\right)\left(\frac{\hat{q}\left(k ; \mathbf{a}_{\mathbf{2}}\right)}{k}\right)^{1-\beta}-\sigma(\delta+\rho)+\delta\left(1+\sigma \tau_{K}\right)+\hat{p}\left(k ; \mathbf{a}_{\mathbf{1}}\right)\right\} \\
& \times \hat{p}\left(k ; \mathbf{a}_{\mathbf{1}}\right)=0 \\
R_{2}\left(k ; \mathbf{a}_{\mathbf{1}}, \mathbf{a}_{\mathbf{2}}\right)= & \hat{q}^{\prime}\left(k ; \mathbf{a}_{\mathbf{2}}\right)\left\{(1-\kappa)\left(\frac{\hat{q}\left(k ; \mathbf{a}_{\mathbf{2}}\right)}{k}\right)^{1-\beta}-\delta-\hat{p}\left(k ; \mathbf{a}_{\mathbf{1}}\right)-\left(1+\frac{\gamma}{1-\beta}\right) \phi(1-l)\right\} k \\
& -\left\{\left(\tau_{K}-\kappa\right)\left(\frac{\hat{q}\left(k ; \mathbf{a}_{\mathbf{2}}\right)}{k}\right)^{1-\beta}+\frac{\delta\left(1-\tau_{K}\right)}{\beta}+\frac{\phi}{\beta} \frac{1-\tau_{L}}{1-\tau_{L}-\tau}-\delta\right. \\
& \left.-\hat{p}\left(k ; \mathbf{a}_{\mathbf{1}}\right)+\phi\left(\frac{\gamma}{\beta}-1\right)(1-l)\right\} \hat{q}\left(k ; \mathbf{a}_{\mathbf{2}}\right)=0 .
\end{aligned}
$$

Calibration of the model To study the effects of fiscal policies in the model, we have to calibrate ${ }^{4}$ the values of the model parameters. We use values for USA which have been used often in related literature. The capital income share $\beta$ is specified to be between 0.25 to $1 / 3$ and we choose the value 0.3. Following Mulligan and Sala-i-Martin (1993), the value of intertemporal elasticity of substitution $\sigma$ is 0.5 (the degree of risk aversion $\theta$ is 2). The rate of depreciation $\delta$ is set to be 10 percent. Since we assume that the before tax real interest rate is 6 percent we can $\operatorname{see}^{5}$ that the coefficient for the productivity of human capital $\phi$ should also be equal to 0.06 (in the case of an absence of externalities.) If we assume that the economy grows at 2 percent per year then we can compute the rate of time preference $\rho=r-g / \sigma$ as 0.02 .

Now we can use any computer implementation of the MWR method to compute a

\footnotetext{
${ }^{4}$ The calibration is a methodology useful for aggregate models to be consistent with existing microeconomic and macroeconomic evidence (see eg Mehra and Prescott (1985)).

${ }^{5}\left(F_{K}\right)_{s s}=\delta+\phi \frac{1-\beta+(2-\sigma) \gamma}{1-\beta+(1-\sigma) \gamma}-\frac{\gamma \rho}{1-\beta+(1-\sigma) \gamma}$ and $\left(F_{K}\right)_{s s}=\delta+\phi$ when $\gamma=0$
} 
numerical solution of the problem ${ }^{6}$. Having prepared the implementation of the problem, we can suggest and perform several fiscal policy experiments, based on different values of tax rate parameters and assess their impacts on the development of the economy.

To demonstrate some model results we suggest the following three experiments. All these experiments have the presence of externality factor $\gamma$ in common $(\gamma=0.4)$. Experiment 1 assumes the benchmark undistorted competitive economy with no taxation and subsidization. Experiment 2 supposes a command economy, i.e. an economy which gives social optimum ${ }^{7}$. Experiment $\mathbf{3}$ is given by the competitive economy with the education subsidy rate $(\tau=0.1)$.

To obtain the solution of the model for these experiments we need to follow two steps. In the first step we use the MWR method to compute an approximation of policy functions and based on this knowledge we simulate the behaviour of model variables in the second step. Obtained transitional dynamics of these three experiments are demonstrated in Fig. 1 and 2. Through comparison of these experiments we can see that in an economy with initially more abundant physical than human capital it is optimal to decrease the level of physical capital much more rapidly (see Fig. 1b) even at the cost of a big decline in consumption during some period (Fig. 1c). The long-run growth rates of all variables, except the working time that has zero growth in the long-run, exhibit higher values (see Fig. 2) for a command economy. Fig. 1 and 2 also show that education subsidies can improve the behaviour of the competitive economy and increase the long-run growth rates.

\footnotetext{
${ }^{6} \mathrm{I}$ used PROJEC which is an implementation of the MWR method in the form of a library module in GAUSS (Kejak (1999)). See the Appendix.

${ }^{7}$ Because of the existence of externalities, the social optimum and the decentralized equilibrium do not coincide. Therefore, we cannot use equations (12)-(14) (more specifically equation (14) must be changed) to specify the TPBV problem for the social optimum (details can be found in (Kejak (1995)).
} 


\section{Summary}

In this paper I discussed the principle of Minimum Weighted Residual Methods. Attention was mainly devoted to one special class of the MWR methods: the orthogonal collocation method with the Chebyshev polynomial basis. Then I demonstrated the application of the MWR methods in a modified Lucas endogenous growth model. Results of the numerical solution to policy model experiments using toolbox PROJEC in GAUSS are finally presented.

\section{Acknowledgements}

I wish to thank the participants of the 17th IFIP Conference on System Modelling and Optimization for useful comments. The usual disclaimer applies.

\section{References}

[1] Fletcher, Clive A. J. (1988) Computational Techniques for Fluid Dynamics 1. SpringerVerlag, New York.

[2] Judd, Kenneth L. (1992) Projection Methods for Solving Aggregate Growth Models. Journal of Economic Theory 58, 410-452.

[3] Judd, Kenneth L. (1998) Numerical Methods in Economics. MIT Press, Boston.

[4] Kejak, Michal (1995) Application of Projection Method in Model of Endogenous Growth. Economics Working Paper Series, No. 7, Institute for Advanced Studies, Vienna.

[5] Kejak, Michal (2000) A User's Guide to the Projection Method in GAUSS. Discussion Paper, CERGE-EI, Prague. 
[6] Lucas, Robert E. Jr. (1988) On the Mechanics of Economic Development. Journal of Monetary Economics 22, 3-42.

[7] Mulligan, Casey B. and Sala-i-Martin Xavier (1993) Transitional dynamics in twosector models of endogenous growth. Quarterly Journal of Economics CVIII, 739-773. 

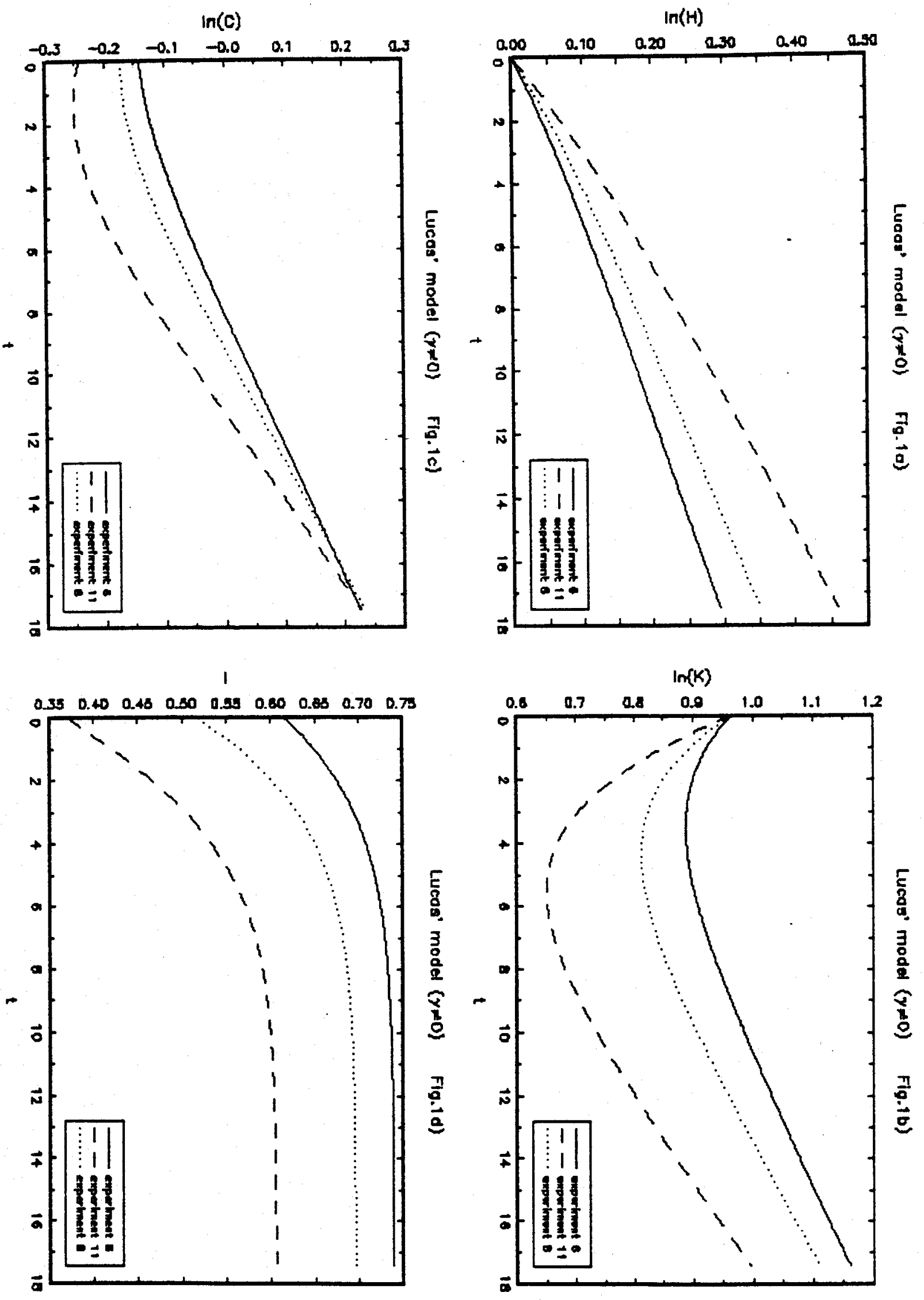
$\because$
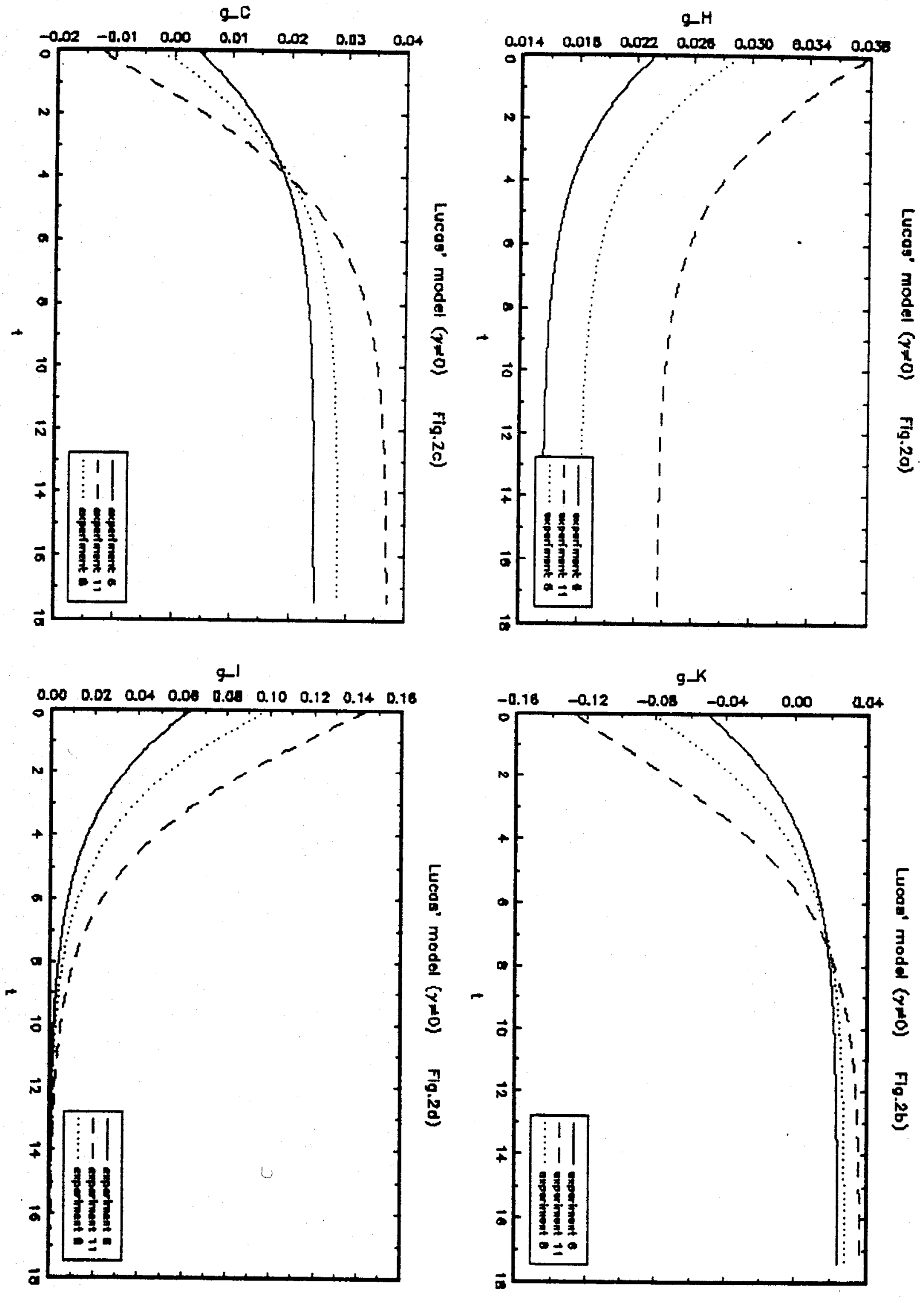


\section{Appendix. Application of PROJEC: GAOSS code}

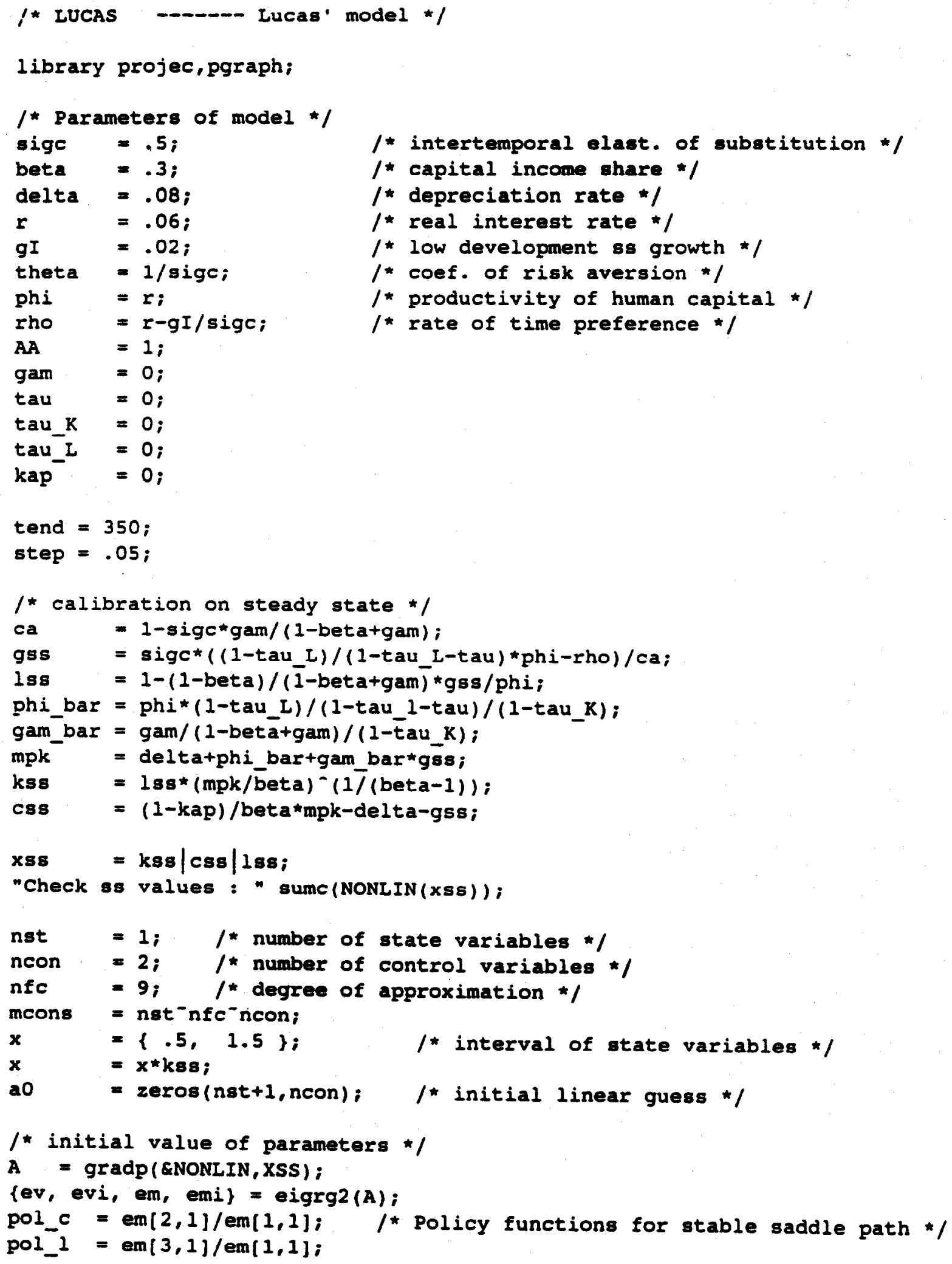




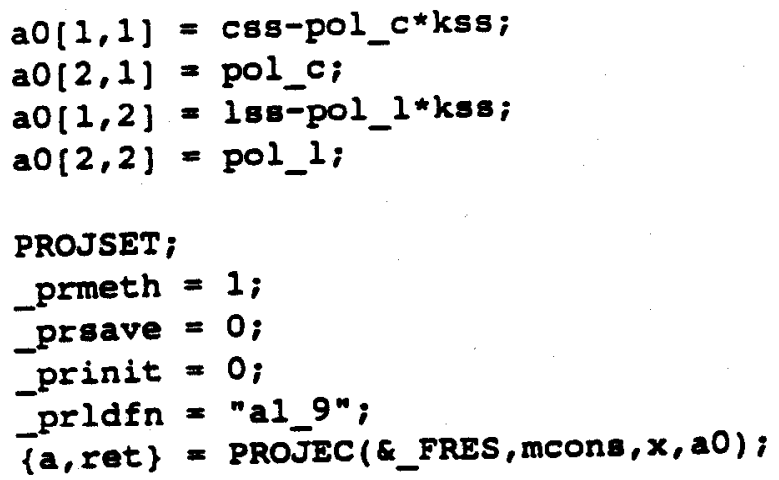

proc NONLIN $(X)$;

local K,C,L, Kdot, $\operatorname{Cdot}, L_{\text {dot, }} F_{-} K_{\text {; }}$

$$
\begin{array}{ll}
\mathrm{K} & =\mathrm{X}[1] ; \\
\mathrm{C} & =\mathrm{X}[2] ; \\
\mathrm{L} & =\mathrm{X}[3] ; \\
\mathrm{F}_{\mathrm{K}} & =(\mathrm{K} / \mathrm{L})-(\operatorname{bet} \mathrm{a}-1) ;
\end{array}
$$

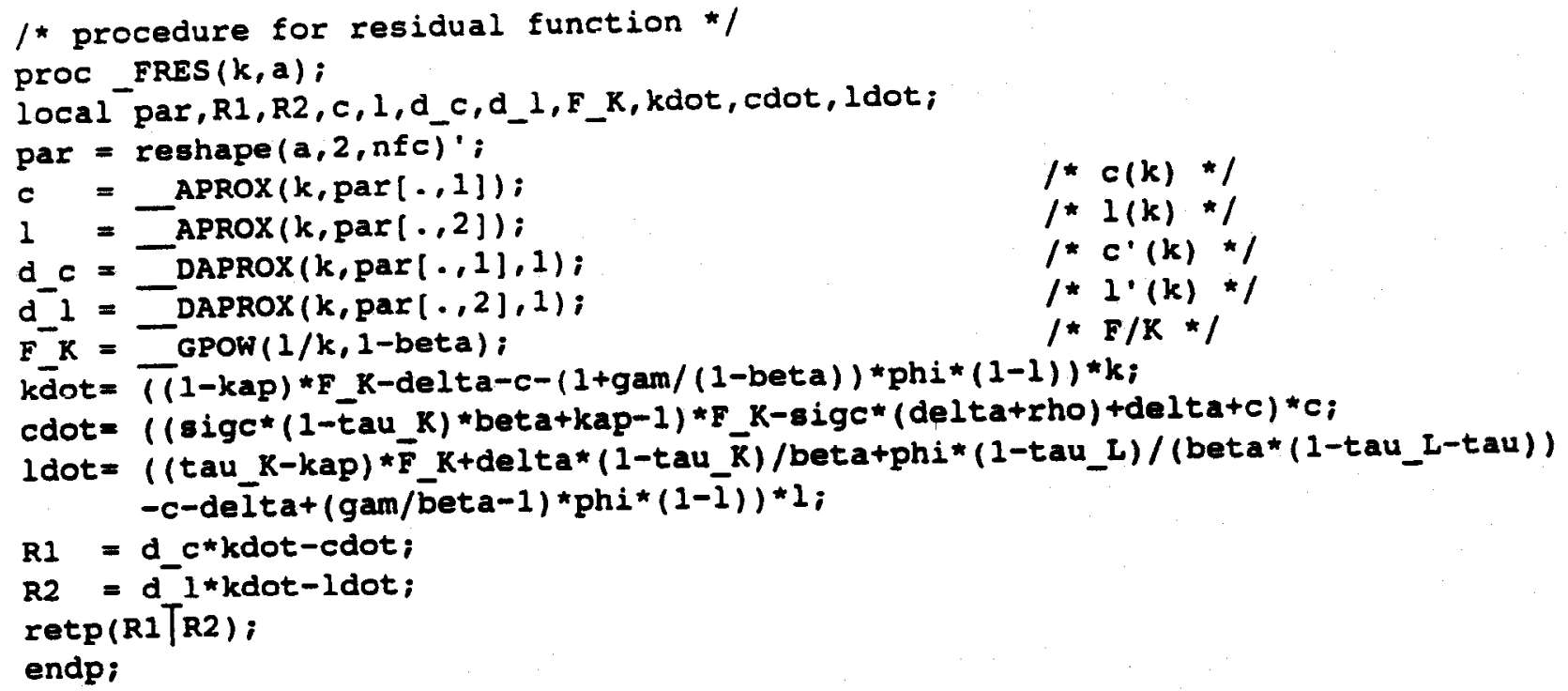

